\title{
Colestasias Familiares: Una Causa Común de Hiperbilirrubinemia Conjugada
}

\author{
MARCELA GODOY P. ${ }^{1}$, HUMBERTO SORIANO B. ${ }^{2}$ \\ 1. Servicio de Pediatría. Unidad de Gastroenterología Pediátrica. Complejo Hospitalario San Borja Arriarán. \\ 2. Unidad de Gastroenterología y Nutrición Pediátrica. Departamento de Pediatría. Facultad de Medicina. \\ Pontificia Universidad Católica de Chile.
}

\section{ABSTRACT \\ Familial Cholestasis: A Comon Cause of Conjugated Hyperbilurubinemia}

Cholestasis is defined as a disorder affecting the production of bile resulting in the retention of its components in the liver and blood. In children, this disorder is almost always due to genetic alterations. Functionally, cholestasis may be the result of hepatic failure to secrete bile due to decrease in transport, synthesis or biliary obstruction. Extrahepatic cholestasis may be caused by biliary atresia and other obstructions of the bile ducts. Intrahepatic cholestasis may be the result of several disorders including progressive familial intrahepatic cholestasis (PFIC) types 1, 2 and 3, an autosomal recessive disease due to mutations in the genes $\mathrm{ATP} 8 \mathrm{~B} 1, \mathrm{ABCB} 11$ and $\mathrm{ABCB} 4$ respectively. Pathophysiology and clinical presentation of this disease are now well understood. Clinically, these patients may present with jaundice, itching, anorexia, and generally unwell. Laboratory tests may disclose conjugated bilirubin over $1 \mathrm{mg} / \mathrm{dl}$ or larger than $20 \%$ of total bilirubin. Ursodeoxycholic acid, cholestiramine and biliary diversion may help in some of these conditions. Ongoing research into the mechanisms of genetic cholestasis could be key to therapy.

(Key words: Cholestasis, progressive familial intrahepatic cholestasis, genetic cholestasis syndromes, hepatobiliary transport).

Rev Chil Pediatr 2009; 80 (4): 367-376

\section{RESUMEN}

La Colestasia corresponde a un trastorno en la formación y excreción de la bilis que provoca retención de sus componentes y daño en hígado y sangre. La colestasia en el niño casi siempre se debe a una alteración hepática secundaria a causas ahora mayormente conocidas a nivel molecular. Desde el punto de vista funcional la colestasia resulta de una insuficiencia secretora del hígado debido a una disminución del flujo biliar por falla en los procesos de transporte o síntesis o a una obstrucción de la vía biliar. La colestasia extrahepática incluye la atresia de vías biliares y otras obstrucciones de la vía biliar. La colestasia intrahepática incluye las colestasias progresivas familiares PFIC 1, 2 y 3 causadas por fallas en los genes ATP8B1, ABCB11 y ABCB4 respectivamente. Clínicamente pueden presentarse con ictericia, prurito,

Trabajo recibido el 09 de diciembre de 2008, aceptado para publicación el 18 de mayo de 2009.

Correspondencia a:

Dr. Humberto Soriano B.

E-mail: hsoriano@med.puc.cl 
anorexia y compromiso del estado general. Desde el punto de vista del laboratorio las enfermedades colestásicas se caracterizan por hiperbilirrubinemia conjugada mayor a $1 \mathrm{mg} / \mathrm{dl}$ o mayor a $20 \%$ de bilirrubina total.

(Palabras clave: Colestasia, colestasia intrahepática familiar progresiva, síndromes colestásicos genéticos, transportador hepatobiliar).

Rev Chil Pediatr 2009; 80 (4): 367-376

\section{Introducción}

La Colestasia se clasifica en extrahepática causada por atresia de vías biliares, quiste de colédoco u otras obstrucciones e intrahepática (tabla 1) $)^{1,2}$ causada por Colestasia intrahepática familiar progresiva (PFIC) tipo 1, 2 ó 3 y colestasia intrahepática recurrente benigna (BRIC); y otras patologías que resultan en colestasia tales como Fibrosis quística, déficit de Alfa1 antitripsina, hemocromatosis neonatal, hipotiroidismo y tirosinemia. Las causas de colestasia en pediatría, sus síntomas clínicos, diagnóstico y tratamiento se resumen en la tabla $2^{3-6}$.

Las PFIC es un grupo de colestasias crónicas de inicio precoz en niños que son la causa más frecuente de colestasia intrahepática y que en general progresan a cirrosis hepática. La primera descripción fue la Enfermedad de Byler en la comunidad Amish en descendientes directos de Jacob Byler y Nancy Kauffman ${ }^{7,8}$, en el condado de Lancaster, cerca de Filadelfia, EE.UU. Posteriormente, se ve que esta enfermedad puede presentarse en otro grupo étnico de niños que no pertenecían a la comunidad Amish por lo que se denomina Síndrome de Byler 9 . Actualmente, se prefiere la nomenclatura PFIC1, 2 ó 3 según el gen afectado ${ }^{10-12}$. Clave para el diagnóstico de las colestasias por PFIC 1 y 2 es que la hiperbilirrubinemia conjugada se acompaña de GGT (Gamma Glutamil Transpeptidasa) normal o baja porque no hay daño de conductos biliares. Sin embargo, en la PFIC 3, el mecanismo del daño es por falla en el trasporte biliar de los fosfolípidos protectores del daño por acción detergente de la bilis lo que resulta en daño de conductillos biliares y elevación importante de la GGT.

Estas diferencias clínicas llevaron al estudio molecular de las causas de estas enfermedades $\mathrm{y}$ en los últimos 12 años se han clonado y caracterizado los genes y transportadores responsables de estos defectos que causan colestasia. Específicamente los subgrupos se han nombrado como PFIC 1/BRIC, PFIC 2 y PFIC 3 o por la consecuencias de la mutaciones descritas: FIC1 (familial intrahepatic cholestasis 1), BSEP (bile salt export pump) y MDR3 (multi drug resistance regulator 3$)^{1,8,10,12-14}$.

A continuación se describen los diferentes tipos de colestasias intrahepáticas familiares (tabla 3$)^{9,15}$.

\section{Colestasia Intrahepática Familiar Progresiva Tipo 1 (PFIC1)}

Se caracteriza por presentarse con ictericia en neonatos de 1 mes a 2,5 meses y con diarrea de inicio entre los 5 a 8 meses. La PFIC1 tiene además manifestaciones extrahepáticas tales como síndrome de malabsorción, pancreatitis y nefrolitiasis. Los exámenes de laboratorio se caracterizan por bilirrubinemia total de hasta 14 $\mathrm{mg} / \mathrm{dl}$ a $17 \mathrm{mg} / \mathrm{dl}$ y de predominio conjugada, transaminasas 2 veces el valor normal, GGT normal o disminuida, colesterol normal, elevación de las sales biliares séricas promedio de 168 umol $(\mathrm{N}<15$ umol) y disminución de la concentración de sales biliares en bilis a 3000 umol ( $\mathrm{N}>10000$ umol) ${ }^{14-16}$.

En la PFIC 1 el gen afectado es ATP8/ FIC1 en el cromosoma 18q21-q 22 $2^{8,14,17}$ FIC1 es una proteína integral de la membrana del canalículo biliar. FIC1 si bien controla la secreción biliar no es un transportador de ácidos biliares. En PFIC1 los mecanismos de inhibición de la secreción biliar hepática todavía se están estudiando, pero serían por el efecto de FIC1 en otros genes. Las manifestaciones extrahepáticas podrían ser explicadas porque FIC1 se expresa además del hígado en otros tejidos, como páncreas, estómago, intestino delgado y riñón ${ }^{18}$. Se ha descrito que los pacientes 
A. Desordenes de transportadores de membrana y secreción

1) Desordenes de secreción canalicular

a) Transportadores ácido biliar - Deficiencia BSEP

- Persistente progresiva (PFIC2)

- Recurrente benigna (BRIC2) $(10,11)$

b) Tansporte fosfolípido-Deficiencia MDR3 (PFIC3)

c) Transporte Ion cloro - Fibrosis quística (CFTR)

2) Desordenes complejos o multiorgánicos

a) Deficiencia del gen FIC1

- Persistente progresiva (PFIC1)

- Recurrente, benigna (BRIC1)

b) Colangitis esclerosante neonatal

c) Artrogriposis

- Disfunción renal

- Síndrome colestásico

B. Desordenes de biosíntesis y conjugación de ácidos biliares

1) Deficiencia de 3-oxo-4 esteroide $5 B$ reductasa

2) Deficiencia 3 B-Hidroxilasa 5 - C27- Dehidrogenasa esferoidal isomerasa

3) Deficiencia Oxysterol 7\& Hidroxilasa

C. Desordenes de embriogénesis y morfológicos

1) Síndrome Alagille - ausencia de conductillos biliares

2) ARPKD Enfermedad poliquística renal autosómica recesiva. (Antiguamente Síndrome de Caroli)

Malformación de la placa ductal en límite de endoderma y mesoderma

3) ADPLD Enfermedad poliquística hepática autosómica dominante. Similar a C2, pero de presentación más tardía

D. No clasificada-Mecanismo desconocido

Abreviaturas: BSEP (bile salt export pump); PFIC (progressive familial intrahepatic cholestasis); BRIC (benign recurrent intrahepatic cholestasis); MDR3 (multi drug resistance regulator); CFTR (cystic fibrosis transmembrane conductance regulator). Modificada de Balistreri-Shneider ${ }^{2,15}$.

que han requerido trasplante hepático continúan con diarrea lo que sugiere un rol del transportador FIC1 a nivel intestinal. Una teoría para explicarlo sería que el defecto FIC1 en intestino altera el ciclo enterohepático de sales biliares. En ratones homocigotos para la mutación G308V en ATP8B1, que es la misma que presentan los niños con PFIC1 se evidenció desregulación del transportador ileal ASBT (apical sodium bile salt transporter), originando una alta absorción intestinal de ácidos biliares con los que fueron alimentados ${ }^{8,18,19}$. En la biopsia hepática de PFIC1 se observa colestasia intracanalicular y en la microscopia electrónica bilis granular típica de Byler lo que es diagnósti$\mathrm{co}^{15}$.

El tipo de mutación se correlacionan en general con la severidad de la presentación clínica, lo que podría explicar la presentación clínica como PFIC1 o BRIC ${ }^{19}$.

Es posible realizar diagnóstico prenatal de enfermedad colestásica hereditaria (PFIC 1-3 y Síndrome de Alagille) mediante screening de mutaciones en DNA obtenido de vellosidades coriónicas y muestras de amniocitos cultiva$\operatorname{dos}^{20}$.

Existe además la posibilidad de estudio mediante gene chip para diagnóstico de mutaciones en pacientes con síndromes colestásicos intrahepáticos hereditarios además de PFIC 1,2 y 3, Síndrome de Alagille y Déficit de Alfa 1 antitripsina ${ }^{21}$.

\section{Colestasia Intrahepática Recurrente Benigna (BRIC)}

Fue descrita por primera vez por Summerskill and Walshe en $1959^{19,22}$. Se han publicado más de 100 casos en el mundo ${ }^{23}$. Al igual que en la PFIC1, el gen involucrado en su patogenia es el FIC1, pero en estos individuos tiene penetrancia variable ${ }^{9}$. Además se ha descrito BRIC2 asociado con mutaciones de $\mathrm{BSEP}^{24,25}$. La modu- 
Tabla 2. Enfermedades Colestásicas: Clínica, diagnóstico y tratamiento

\begin{tabular}{|c|c|c|c|c|}
\hline Enfermedad & Clínica & Prueba hepática & Examen diagnóstico & Tratamiento \\
\hline Atresia de vías biliares & $\begin{array}{l}\text { Ictericia } \\
\text { Acolia } \\
\text { Coluria }\end{array}$ & $\begin{array}{l}\text { Bili T } 16 \pm 3 \mathrm{mg} / \mathrm{dl} \\
\text { Bili d } 5 \pm 3 \mathrm{mg} / \mathrm{dl} \\
\text { GGT } 350 \pm 330 \mathrm{IU} / \mathrm{l}\end{array}$ & $\begin{array}{l}\text { Ecografía abdominal } \\
\text { Biopsia hepática } \\
\text { HIDA } \\
\text { Colangio intraoperatoria }\end{array}$ & $\begin{array}{l}\text { Kassai }<6 \text { - } 8 \text { semanas } \\
\text { Tx hepático }\end{array}$ \\
\hline Quiste de colédoco & Igual a 1 & Igual a 1 & Ecografía abdominal & $\begin{array}{l}\text { Y de Roux } \\
\text { Resección completa } \\
\text { (Carcinoma biliar) }\end{array}$ \\
\hline Síndorme de Alagille & $\begin{array}{l}\text { Facies típica } \\
\text { Prurito } \\
\text { Xantomas } \\
\text { Cardiopatía }\end{array}$ & $\begin{array}{l}\text { GGT elevada } \\
\text { Ácidos biliares elevados }\end{array}$ & $\begin{array}{l}\text { Rx Columna } \\
\text { Ecocardiograma } \\
\text { Ex. Oftalmológico } \\
\text { Bx Hepática } \\
\text { (sin conductos biliares) }\end{array}$ & $\begin{array}{l}\text { URSO } \\
\text { Colestiramina } \\
\text { Tx Hepático }\end{array}$ \\
\hline Fibrosis quística & $\begin{array}{l}\text { RN igual a } 1 \\
\text { Mayores cirrosis biliar } \\
\text { focal }\end{array}$ & & $\begin{array}{l}\text { Electrolitos en sudor } \\
\text { Esteatocrito } \\
\text { Gen CFTR }\end{array}$ & $\begin{array}{l}\text { Enzimas } \\
\text { URSO }\end{array}$ \\
\hline Déficit Alfa-1-Antitripsina & $\begin{array}{l}\text { Ictericia } \\
\text { Hepatoesplenomegalia } \\
\text { Síntomas pulmonares } \\
\text { Tos, asma }\end{array}$ & $\begin{array}{l}\text { GGT elevada } \\
\text { Ac. Biliares elevados } \\
\text { Coagulopatía }\end{array}$ & $\begin{array}{l}\text { Alfa -1- antitripsina } \\
\text { Biopsia hepática } \\
\text { (acúmulo A1AT en } \\
\text { hepatocito) }\end{array}$ & Tx hepático si es severo \\
\hline Tirosinemia & $\begin{array}{l}\text { Ictericia } \\
\text { Insuficiencia hepática }\end{array}$ & $\begin{array}{l}\text { Alfa fetoproteína } \\
>400000 \text { ug/l }\end{array}$ & Succinilacetona en orina & NTBC \\
\hline $\begin{array}{l}\text { Hemocromatosis } \\
\text { Neonatal }\end{array}$ & Insuficiencia hepática & Ferritina $>800 \mathrm{ng} / \mathrm{ml}$ & $\begin{array}{l}\text { Biopsia glándula salival } \\
\text { labio }\end{array}$ & $\begin{array}{l}\text { Prevención en embarazo } \\
\text { Gamma EV } \\
\text { CAO }\end{array}$ \\
\hline $\begin{array}{l}\text { Déficit síntesis } \\
\text { Ác. biliares }\end{array}$ & $\begin{array}{l}\text { Hiperbilirrubinemia } \\
\text { conjugada }\end{array}$ & Ác. biliares disminuidos & $\begin{array}{l}\text { Espectrometría de masa } \\
\text { en orina }\end{array}$ & $\begin{array}{l}\text { Existe tratamiento } \\
\text { específico dependiendo } \\
\text { del déficit }\end{array}$ \\
\hline Hipotiroidismo & $\begin{array}{l}\text { Hipotonía } \\
\text { Retraso crecimiento y } \\
\text { desarrollo } \\
\text { Hernia umbilical }\end{array}$ & & $\begin{array}{l}\text { T4 libre } \\
\text { TSH }\end{array}$ & Levotiroxina \\
\hline Galactosemia & $\begin{array}{l}\text { Catarata } \\
\text { Hipoglicemia } \\
\text { Hepatoesplenomegalia } \\
\text { Hipotonía }\end{array}$ & & $\begin{array}{l}\text { Galactosa total } \\
\text { Galactosa 1-P } \\
\text { uridiltransferasa } \\
\text { (screening neonatal } \\
\text { ampliado) }\end{array}$ & Dieta sin galactosa \\
\hline
\end{tabular}

PFIC 1, 2 y 3 se discute más adelante en tabla 3.

GGT: Gamma glutamil transferasa; HIDA: Hepatic Imido Diacetic Acid; NTBC (2-(2-nitro-4-fluoromethylbenzoyl)-1,3-cyclohexanedione); URSO: Ácido Ursodeoxicolico; CAO: cocktail antioxidante.

lación del fenotipo por otros genes asociados es materia de activa investigación. En BRIC la edad de presentación es variable de niño a adulto (5 meses a 47 años). Esta enfermedad se caracteriza por episodios recurrentes de hiperbilirrubinemia conjugada con valores hasta 10 veces el valor normal ${ }^{23}$ desencadenada por virosis en niños y adultos. La BRIC presenta recurrencia estacional en muchos pacientes (primavera, verano) cada 2 años. La duración del episodio puede ser de 2 semanas a 18 meses (promedio 3 meses), pudiendo permanecer asintomático por un período variable (1 mes a 33 años). La mayoría de estos pacientes presenta prurito aunque el $25 \%$ puede no tenerlo. En la evaluación del primer episodio se debe incluir estudios serológicos para excluir hepatitis viral aguda y crónica así como también otras causas de enfermedad hepática crónica. Además es necesario suspender todos los medicamentos que pudieran causar colestasia como Acido clavulánico, azatioprina, eritromicina y 
Tabla 3. Comparación de PFIC 1, 2 ,3 y BRIC

\begin{tabular}{|c|c|c|c|c|}
\hline & PFIC1 & PFIC2 & PFIC3 & BRIC \\
\hline Transportador & $\mathrm{FIC1}$ & BSEP & MDR3 & $\mathrm{FIC1}$ \\
\hline Herencia & $\begin{array}{l}\text { Autosómica } \\
\text { Recesiva }\end{array}$ & $\begin{array}{l}\text { Autosómica } \\
\text { Recesiva }\end{array}$ & $\begin{array}{l}\text { Autosómica } \\
\text { Recesiva }\end{array}$ & $\begin{array}{l}\text { Autosómica } \\
\text { Recesiva }\end{array}$ \\
\hline Gen & ATP8B1 & $\mathrm{ABCB} 11$ & $\mathrm{ABCB} 4$ & ATP8B1 \\
\hline Cromosoma & $18 q 21-q 22$ & $2 q 24-31$ & $7 q 21$ & $18 q 21-22$ \\
\hline $\begin{array}{l}\text { Clínica } \\
\text { Sint. extrahepático } \\
\text { Prurito }\end{array}$ & $\begin{array}{l}\text { Si } \\
\text { Intenso }\end{array}$ & $\begin{array}{l}\text { No } \\
\text { Intenso }\end{array}$ & $\begin{array}{l}\text { No } \\
\text { Leve a moderado }\end{array}$ & $\begin{array}{l}\text { No } \\
\text { Ausente 25\% }\end{array}$ \\
\hline $\begin{array}{l}\text { Laboratorio } \\
\text { GGT } \\
\text { Colesterol } \\
\text { Ác. biliares séricos }\end{array}$ & $\begin{array}{l}\text { Normal } \\
\text { Normal } \\
\text { Elevado }\end{array}$ & $\begin{array}{l}\text { Normal } \\
\text { Normal } \\
\text { Elevado }\end{array}$ & $\begin{array}{l}\text { Elevada } \\
\text { Normal o elevado } \\
\text { Elevado }\end{array}$ & $\begin{array}{l}\text { Normal } \\
\text { Normal } \\
\text { Elevado (recaída) }\end{array}$ \\
\hline Histología & Bilis de Byler & Hepatitis células gigantes & Proliferación ductal & Colestasia centrolobulillar \\
\hline Evolución & Cirrosis & Cirrosis & Cirrosis & Benigna \\
\hline
\end{tabular}

clorpromazina $^{26-28}$. Es fundamental realizar ecografía abdominal para excluir patologías biliares obstructivas con dilatación de la vía biliar.

En adolescentes o adultos la colangioresonancia o la colangiografía retrograda endoscópica se pueden utilizar para excluir colangitis esclerosante $\mathrm{u}$ otras causas de alteraciones estructurales de árbol biliar. Es importante realizar una biopsia hepática para precisar el diagnóstico y descartar otras patologías ${ }^{23}$. La biopsia hepática muestra una colestasia centrolobulillar sin lesión hepática o ductular a pesar de que puede observarse un leve infiltrado inflamatorio $^{19,23,29}$.

La diferencia entre BRIC y las colestasias progresivas (PFIC 1, 2 y 3 ) es que estas últimas progresan hasta llegar a cirrosis 9,13 . Es muy raro que el BRIC progrese a fibrosis o requiera trasplante hepático ${ }^{30}$.

\section{Colestasia Intrahepática Familiar Progresiva Tipo 2 (PFIC2)}

En PFIC2 la clínica es similar a la que presentan los pacientes con PFIC1. La edad de presentación es principalmente en neonatos (rango: 1 mes a 5 años) ${ }^{31}$. Presentan ictericia con hiperbilirrubinemia total en rango de 7 a 11 $\mathrm{mg} / \mathrm{dl}$, bilirrubinemia directa en rango de 4,5 a $9,5 \mathrm{mg} / \mathrm{dl}$. ALT en rango de 100 a $200 \mathrm{IU} / \mathrm{l}$. Al igual que en PFIC1 la GGT y el colesterol son normales. En la Ecografía abdominal pueden tener hepatoesplenomegalia leve y litiasis bi$\operatorname{liar}^{18,32}$. La diferencia radica en que tienen una evolución más rápida a cirrosis e insuficiencia hepática. En PFIC2 no se han encontrado otros órganos afectados. Posterior al trasplante hepático no desarrollan diarrea y recuperan la curva estatural ${ }^{18}$.

El gen afectado es ABCB11/BSEP en cromosoma $2 \mathrm{q} 24^{8}$. BSEP participa en el transporte de ácidos biliares hacia la bilis a nivel de la membrana canalicular del hepatocito. La biopsia hepática demuestra transformación gigante celular similar a la descrita en hepatitis neonatal ${ }^{33}$.

\section{Colestasia Intrahepática Familiar Progresiva Tipo 3 (PFIC 3)}

La presentación clínica de PFIC3 es diferente a PFIC1 y 2, la edad de presentación es de 1 mes a 20 años ${ }^{31,34}$. Sólo un tercio de los casos se diagnostican antes del año de vida y muy rara vez su debut es como colestasia neonatal en cuyo caso es parte del diagnóstico diferencial de la atresia de vías biliares, la causa más frecuente de colestasia. La mayoría de los niños son diagnosticados por hepatoesplenomegalia o por complicaciones de su cirrosis hepática durante la infancia o adoles- 
cencia $^{18}$. Dentro de los exámenes de laboratorio tienen hiperbilirrubinemia directa en rango de $1,2 \mathrm{mg} / \mathrm{dl}$ a $1,8 \mathrm{mg} / \mathrm{dl}$, ALT rango de $171 \mathrm{IU} / 1$ a $250 \mathrm{IU} / 1(\mathrm{~N}<40 \mathrm{IU} / 1)$ y GGT en rango de 280 IU/1 a $360 \mathrm{IU} / 1(\mathrm{~N}<25 \mathrm{IU} / 1)^{34}$. La GGT elevada en PFIC3 sirve para diferenciarla de la PFIC1, PFIC2 y BRIC en los que la GGT es normal o baja. La GGT es elevada en PFIC3 pues es la única que causa daño endotelial de los conductos y conductillos biliares ${ }^{9}$.

En PFIC3 el gen afectado es ABCB4/ MDR3 en cromosoma 7q21 ${ }^{9}$. El MDR3 es el transportador de fosfolípidos hacia la bilis. Los fosfolípidos cumplen la función de solubilizar las sales biliares y proteger el epitelio ductal ${ }^{11,18}$. En la biopsia hepática se demuestra proliferación ductal acompañada de fibrosis y cirrosis ${ }^{33}$. En pacientes o familiares de pacientes con PFIC 3 se han reportado litiasis biliar, episodios de colestasia del embarazo y litiasis intrahepática $^{34,35}$.

\section{Fisiopatología de PFIC 1, 2, 3 y BRIC}

El componente principal de la bilis son los ácidos biliares que son sintetizados en el hígado a partir del colesterol; son tóxicos para las membranas por lo que deben ser transportados unidos a fosfolípidos y colesterol ${ }^{18}$. Los ácidos biliares a nivel de la membrana sinusoidal del hepatocito son transportados por NTCP $(\mathrm{Na}+$ taurocholate co-transporting polypeptide) que necesita bomba $\mathrm{Na} / \mathrm{K}$ ATPasa para mantener el gradiente eléctrico intracelular adecuado y por OATPs (organic anion transporting polypeptides) independiente de la bomba $\mathrm{Na} / \mathrm{K}$ ATPasa. Al llegar a la membrana canalicular son transportados por BSEP (bile salt export pump) a la bilis. El MDR3 (multi drug resistance regulator) es el transportador de fosfolípidos hacia la bilis. Los fosfolípidos cumplen la función de solubilizar las sales biliares y proteger el epitelio ductal ${ }^{11,18,36}$ (figura 1). La regulación de la actividad excretora de bilis se realiza por el sensor fisiológico de sales biliares denominado FXR (farnesoid X receptor) ${ }^{12,17,18,37,38}$. Los ácidos biliares (ácido cólico, ácido deoxicólico y ácido litocólico) activan a FXR y a otros receptores regulando el transporte y biosíntesis de los ácidos biliares y lípidos ${ }^{12,17}$. En el hepatocito FXR induce la expresión de BSEP aumentando la excreción de sales biliares ${ }^{11,14}$ e inhibe la expresión de NTCP con lo cual disminuye la entrada de sal biliar en el polo sinusoidal del hepatocito. Además, FXR inhibe la expresión de enzimas involucradas en la síntesis de ácidos biliares a partir del colesterol, es decir, neosíntesis $^{14}$. Esta acción la realiza activando SHP (short heterodimer protein o small heterodimer partner), una proteína que en el ileon inhibe la expresión de ASBT (apical sodium bile SALT transporter), transportador que se encarga de la reabsorción de sales biliares que posteriormente llegan al hígado mediante sistema portal. El exacto rol de FIC1 el gen de la PFIC1 es desconocido, aunque estaría relacionado con la reducción en la expresión de $\mathrm{FXR}^{9,19}$ lo que resulta en disminución del flujo biliar con acumulación de ácidos biliares.

\section{Tratamiento de la colestasia}

El tratamiento de la colestasia va a depender de la causa. No todos los casos de colestasia tienen terapia específica. Se debe suplementar con vitaminas liposolubles (A, D, E y K) y calcio en colestasia crónica puesto que la falta

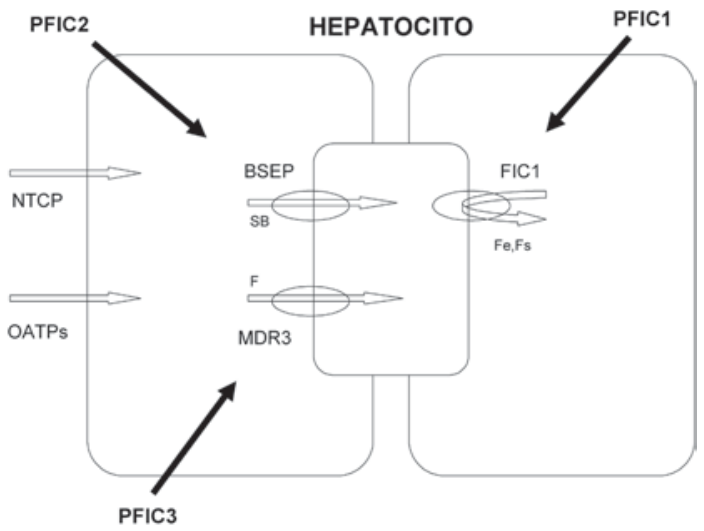

Figura 1. Transportadores de ácidos biliares. Muestra el mecanismo fisiopatológico de los transportadores hepatobiliares involucrados en PFIC 1-3. En la membrana sinusoidal: NTCP (Na+ taurocholate co- transporting polypeptide) y OATPs (organic anion transporting polypeptides) y en la membrana canalicular: BSEP (bile salt export pump), MDR3 (multi drug resistance regulator) y FIC1 (proteína que trasloca aminofosfolípidos). Fe: fosfatidil etanolamina, Fs: fosfatidil serina, SB: sales biliares, F: fosfolípidos. 


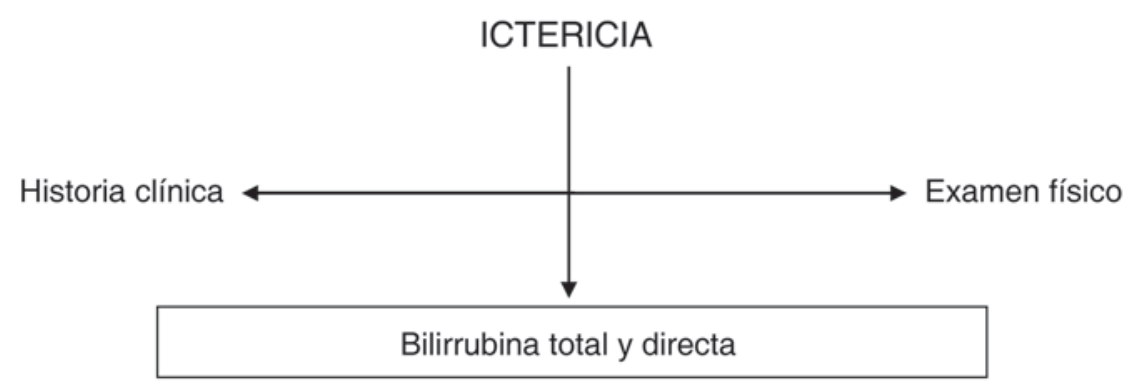

\begin{tabular}{|l|}
\hline Bilirrubina indirecta \\
\hline Lactancia materna \\
Hemólisis \\
Infección \\
Hipotiroidismo \\
Estenosis hipertrófica píloro \\
\hline
\end{tabular}

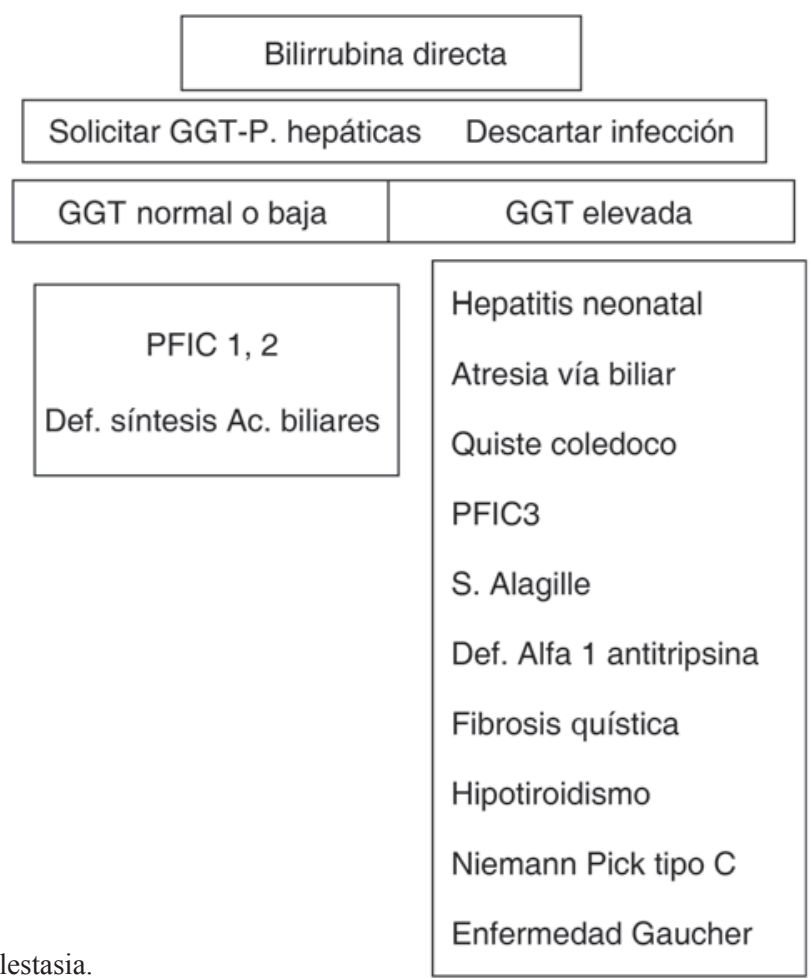

Figura 2. Algoritmo diagnóstico de colestasia.

de bilis resulta en malabsorción de grasa con saponificación que quela el calcio y reduce su absorción. En el tratamiento del prurito se ha utilizado colestiramina en dosis de 2 a 8 g/día ${ }^{20}$ que es una resina de intercambio aniónico que liga sales biliares en el intestino y aumenta su eliminación fecal y disminuye el prurito en PFIC 1 y $2^{20}$. Quizás por efecto de otros genes moduladores, la respuesta al ursodeoxicolico y a la colestiramina es a veces dramática y a veces su uso resulta en empeoramiento de los síntomas por lo que el tratamiento debe ser individual.

\section{Mecanismo de Acción de Ácido Ursodeoxicólico (UDCA)}

UDCA (Bilis de oso usado en medicina china) es ampliamente usado para el tratamiento de una variedad de enfermedades hepáticas colestásicas crónicas. En humanos alcanza el $3 \%$ del total de ácidos biliares. Los primeros reportes de sus efectos beneficiosos fueron publicados en el año 1980 por Leuschner et $\mathrm{al}^{39}$. Dentro de los mecanismos de acción de UDCA se describen la protección de los colangiocitos contra los efectos tóxicos de lo ácidos biliares ${ }^{38}$, estimulación de la secreción 
biliar y de los ácidos biliares hidrofóbicos e inhibición de la apoptosis de los hepatocitos ${ }^{39,40}$. A nivel de los transportadores hepatobiliares el UDCA estimula la expresión de BSEP lo que facilita el transporte de sales biliares a nivel canalicular e inhibe ASBT (apical Na+ dependent bile salt transporter) a nivel intestinal lo que facilita la eliminación de sales biliares del organismo $^{15,38}$. En resumen los efectos de UDCA son: colerético, inmunomodulador, citoprotector y antiapoptótico ${ }^{14}$. La dosis de UDCA en niños con colestasia es $20 \mathrm{mg} / \mathrm{kg} / \mathrm{día}^{41,42}$. Otros reportes sugieren 20 a $30 \mathrm{mg} / \mathrm{kg} / \mathrm{dí}^{39,40}$.

UDCA en PFIC3 sólo funcionaría en pacientes que tengan una mutación missense (actividad residual de la proteína). Los pacientes que no responden al tratamiento con UDCA deben ser considerados para el trasplante hepático ${ }^{9,43}$ o para la derivación biliar.

La dosis adecuada de Ursodeoxicólico en BRIC es desconocida ${ }^{23}$, pero se ha utilizado dosis de $15 \mathrm{mg} / \mathrm{kg} /$ día hasta la desaparición de la clínica y luego $6 \mathrm{mg} / \mathrm{kg} /$ día hasta normalización de parámetros de laboratorio ${ }^{29}$. Su objetivo sería acortar el período de colestasia ${ }^{29}$. El TUDCA (tauroursodeoxycholic acid) se ha utilizado en forma experimental. Estimula la inserción de proteínas transportadoras en la membrana canalicular del hepatocito ${ }^{38,44}$.

El trasplante hepático es el tratamiento más eficaz en PFIC1 y 2 para pacientes con enfermedad progresiva o cirrosis establecida, en general en la primera década, con sobrevida sobre el $80 \%$ a largo plazo. La derivación biliar externa parcial también existe como un tratamiento de respuesta variable para pacientes con PFIC1 y $2^{9}$. En aquellos pacientes con prurito intratable sin respuesta a tratamiento médico la derivación biliar externa parcial tiene efecto importante sobre la colestasia y el crecimiento de los niños, aunque no todos ellos se benefician de la cirugía. Episodios de deshidratación después de efectuada la derivación deben ser considerados ${ }^{45,46}$.

En resumen se han realizado grandes avances en el diagnóstico y biología molecular de la colestasia intrahepática otrora hepatitis neonatal. Estos avances auguran un futuro optimista para el manejo y tratamiento de estas enfermedades de alta morbimortalidad en niños.

\section{Referencias}

1.- Balistreri $W$ : Intrahepatic Cholestasis. J Pediatr Gastroenterol Nutr 2002; 35: S17-S23.

2.- Balistreri W, Bezerra J, Jansen P, Karpen S, Schneider $L$, Suchy F: Intrahepatic Cholestasis:Summary of an American Association for the study of Liver Disease Single-Topic Conference. Hepatology 2005; 42 (1): 222-35.

3.- Tang K, Huang L, Huang Y, et al: Gamma-glutamyl transferase in the diagnosis of biliary atresia. Acta Paediatr Taiwan 2007; 48 (4): 196-200.

4.- García R, Gómez L, García R: Tirosinemia tipo I: dos formas atípicas de presentación clínica. Acta Pediatr Esp 2006; 64: 286-9.

5.- Whitington $P$, Kelly $S$, Ekong $U$ : Neonatal hemochromatosis: fetal liver disease leading to liver failure in the fetus and newborn. Pediatr Transplantation 2005; 9: 640-5.

6.- Bosch A: Classical galactosaemia revisited.J Inherit Metab Dis 2006; 29: 516-25.

7.- Clayton R, Iber F, Ruebner B, Mc Kusick V: Byler's disease: fatal familial intrahepatic cholestasis in an Amish kindred. J Pediatr 1965; 67:1025-8.

8.- Hutchins G, Gollan J: Recent developments in the pathophysiology of Cholestasis. Clin Liver Dis 2004; 8 (1): 1-26.

9.- Harris M, Le Couteur D, Arias I: Progressive familial intrahepatic cholestasis:Genetic disorders of biliary transporters. J Gastroenterol Hepatol 2005; 20: 807 17.

10.- Oude R: Undertanding and controlling hepatobiliary function. Best Pract Res Clin Gastroenterol 2002; 16 (6): 1025-34.

11.- Arrese M, Trauner M: Molecular aspects of bile formation and cholestasis. Trends Mol Med 2003; 9 (12): 558-63.

12.- Keitel V, Budelski M, Warsukulat, et al: Expression and localization of hepatobiliary transport proteins in progressive familial intrahepatic cholestasis. Hepatology 2005; 41: 1160-72.

13.- Klomp L, Vargas J, Van Mil S, et al: Characterization of Mutations in ATP8B1 Associated with Hereditary Cholestasis. Hepatology 2004; 40: 27-38.

14.- Trauner M, Boyer J: Cholestatic Syndromes. Curr Opin Gastroenterol 2003; 19: 216-31.

15.- Alissa F, Jaffe R, Shneider B: Update on progressive Familial Intrahepatic Cholestasis. J Pediatr Gastroenterol Nutr 2008; 46: 241-52.

16.- Lykavieris P, Van Mil S, Cresteil D: Progressive familial 
intrahepatic cholestasis type 1 and extrahepatic features: no catch-up of stature growth, exacerbation of diarrhea and appearance of liver steatosis after liver transplantation. Journal of Hepatology 2003; 39: 44752.

17.- Rutherford A, Pratt D: Cholestasis and Cholestatic Syndromes. Curr Opin Gastroenterol 2006; 22: $209-$ 14.

18.- Hierro L, Jara P: Colestasis infantile y transportadores biliares. Gastroenterol Hepatol 2005; 28 (7): 38895.

19.- Ferenci P, Zollner G, Trauner M: Hepatic Transport Systems. J Gastroenterol Hepatol 2002; 17 suppl: S105-12.

20.- Jung C, Driancourt C, Baussan C, et al: Prenatal Molecular Diagnosis of Inherited Cholestatic Diseases. J Pediatr Gastroenterol Nutr 2007; 44: 453-8.

21.- Liu C, Aronow B, Jegg A, et al: Novel resequency chip customized to diagnose mutations in patients with inherited syndromes of intrahepatic cholestasis. Gastroenterology 2007; 132 (1): 119-26.

22.- Summerskill $W$ : Benign recurrent intrahepatic "obstructive" jaundice. Lancet 1959; 2: 686-9.

23.- Luketic V, Shiffman M: Benign recurrent intrahepatic cholestasis. Clin Liver Dis 2004; 8: 133-49.

24.- Van Mil S, Van Der Woerd W, Van Brugge G, et al: Benign Recurrent Intrahepatic Cholestasis Type 2 is caused by Mutations in ABCB11. Gastroenterology 2004; 127: 379-84.

25.- Kubitz R, Keitel V, Scheuring S, Kohrer K, Haussinger $D$ : Benign Recurrent Intrahepatic Cholestasis Associated with Mutations of the Bile Salt Export Pump. J Clin Gastroenterol 2006; 40: 81-5.

26.- Mohi-ud-din R, Lewis J: Drug and chemical induced cholestasis. Clin Liver Dis 2004; 8: 95-132.

27.- Pauli-Magnus Ch, Meier P: Hepatobiliary transporters and drug induced cholestasis. Hepatology 2006; 44 : 778-87.

28.- Chang C, Schiano T: Drug hepatotoxicity. Aliment Pharmacol Ther 2007; 25 (10): 1135-51.

29.- Nicolás I, Pons J, Vargas A, Gallardo F, Albaladejo A, Nicolas: El tratamiento con ácido ursodexosicólico parece acortar el período de Cholestasis en 2 pacientes con Cholestasis intrahepática benigna. J Gastroenterol Hepatol 2003; 26 (7): 421-3.

30.- Mezey E, Burns C: A case of severe benign intrahepatic cholestasis treated with liver transplantation. Am J Gastroenterol 2002; 97 (2): 475-7.

31.- Pauli-Magnus Ch, Stieger B, Meier Y, Kullak-Ublick $G$, Meier P: Enterohepatic transport of bile salts and genetics of cholestasis. J Hepatol 2005; 43: 342-57.

32.- Nobili V, Giandomenico S, Francalnci P, Callea F, Marcellini $M$, Santorelli $F$ : A new ABCB11 mutation in two Italian children with familial intrahepatic cholestasis. J Gastroenterol 2006; 41: 598-603.

33.- Knisely A, Strautnieks S, Meier Y: Hepatocelular carcinoma in ten children under five years of age with bile salt export pump deficiency. Hepatology 2006; 44 (2): 478-86.

34.- Jacquemin E, Devree M, Cresteil D: The wide spectrum of multidrug resistance 3 deficiency: From neonatal cholestasis to cirrhosis of adulthood. Gastroenterology 2001; 120: 1448-58.

35.- Jansen P, Sturm E: Genetic cholestasis, causes and consequences for hepatobiliary transport. Liver int 2003; 23:315-22.

36.- Jansen P, Muller M, Sturm E: Genes and Cholestasis. Hepatology 2001; 34 (6): 1067-74.

37.- Trauner M, Wagner M, Fickert P, Zollner G: Molecular regulation of Hepatobiliary transport Systems. J Clin Gastroenterol 2005; 39: S111-24.

38.- Geier A, Wagner M, Dietrich C, Trauner M: Principles of hepatic organic anion transporter regulation during cholestasis,inflammation and liver regeneration. Bioch Biophys Acta 2007; 1773: 283-308.

39.- Paumgartner G, Beuers $U$ : Mechanisms of action and therapeutic efficacy of ursodeoxycholic acid in cholestatic liver disease. Clin Liver Dis 2004; 8: 6781.

40.- Paumgartner G, Beuers U: Ursodeoxycholic Acid in Cholestatic Liver Disease: Mechanisms of action and Therapeutic use revisited. Hepatology 2002; 36: 52531.

41.- George R, Stevens A, Berkenbosch JW, Turpin J, Tobias $J$ : Ursodeoxycholic acid in the treatment of cholestasis and hyperbilirubinemia in pediatric intensive care unit patients. South Med J 2002; 95 (11): 12769.

42.- Van de Meeberg P, Houwen R, Sinaasappel M, Heijerman H, Bijleveld C, Vanberge-Henegouwen $G$ : Low-dose versus high-dose ursodeoxycholic acid in cystic fibrosis-related cholestatic liver disease. Results of a randomized study with 1-year follow-up Scand. J Gastroenterol 1997; 32 (4): 369-73.

43.- Espinosa M, Navas V, Blasco J, Sierra C, Barco A: Cholestasis intrahepática familiar progresiva tipo 3. Defecto de MDR3. An Pediatr 2008; 69(2): 18196.

44.- Beuers U, Bilzer M, Chittattu A: Tauroursodeoxycholic acid inserts the apical conjugate export pump, Mrp2, 
SORIANO H. y col.

into canalicular membranes and stimulates organic anion secretion by protein kinase C-dependent mechanisms in cholestatic rat liver. Hepatology 2001; 33: 1206-16.

45.- Ekinci S, Karnak I, Gurakan F: Partial external biliary diversion for the treatment of intractable pruritus in children with progressive familial intrahepatic
Cholestasis: Report of two cases. Surg Today 2008; 38 (8): 726-30.

46.- Arnell H, Bergdahl S, Papadogiannakis N, Nemeth A, Fischler B: Preoperative observations and short-term outcome after partial external biliary diversion in 13 patients with progressive familial intrahepatic Cholestasis. J Pediatr Surg 2008; 43 (7): 1312-20. 